\title{
Energy-resolved secondary-electron emission of candidate beam screen materials for electron cloud mitigation at the Large Hadron Collider
}

\author{
S. Schulte ${ }^{*}$, G. Hartung $\odot$, and J. Kröger $\odot$ \\ Institut für Physik, Technische Universität Ilmenau, D-98693 Ilmenau, Germany \\ M. Himmerlich®, V. Petitø, and M. Taborelli \\ CERN, European Organization for Nuclear Research, CH-1211 Meyrin, Switzerland
}

(Received 17 April 2020; accepted 28 September 2020; published 21 October 2020)

\begin{abstract}
Energy-resolved secondary electron spectroscopy has been performed on air-exposed standard $\mathrm{Cu}$ samples and modified $\mathrm{Cu}$ surfaces that are tested and possibly applied to efficiently suppress electron cloud formation in the high-luminosity upgrade of the Large Hadron Collider at CERN. The $\mathrm{Cu}$ samples comprise pristine oxygen-free, carbon-coated and laser-structured surfaces, which were characterized prior to and after electron irradiation and rare-gas ion bombardment. Secondary-electron and reflected-electron yields measured with low charge dose of the samples exhibit a universal dependence on the energy of the primary impinging electrons. State-of-the-art models can successfully be used to describe the spectroscopic data. The supplied spectral dependence of electron emission and integrated electron yield as well as the derived parametrization can serve as a basis for forthcoming simulations of electron cloud formation and multipacting.
\end{abstract}

DOI: 10.1103/PhysRevAccelBeams.23.103101

\section{INTRODUCTION}

The emission of secondary electrons (SE) from a surface induced by electron irradiation is a common effect used in electron multipliers and detectors $[1,2]$ as well as a specific contrast mechanism in scanning electron microscopes [3-5]. In some applications, this process creates undesirable phenomena such as charge build-up at the surface of spacecrafts and satellites [6,7] or the formation of an electron cloud in particle accelerators [8-12]. The latter is a major challenge for the high-luminosity upgrade of the Large Hadron Collider (LHC) because it provokes significant heat load in the cryogenic sections as well as beam instabilities or even intensity losses [13]. Strategies to reduce the secondary electron yield (SEY), which defines the number of emitted electrons per impinging electron, of relevant vacuum components below the electron multipacting threshold such as amorphous carbon (a-C) coating $[14,15]$ as well as laser-induced surface modification [16-18] have been tested and validated on a laboratory scale. Current developments aim at transferring these technologies to large-scale treatments of LHC units. As an example, a-C coatings are presently under implementation in the Super Proton

\footnotetext{
*stefan.schulte@tu-ilmenau.de

Published by the American Physical Society under the terms of the Creative Commons Attribution 4.0 International license. Further distribution of this work must maintain attribution to the author(s) and the published article's title, journal citation, and DOI.
}

Synchrotron at CERN and in standalone LHC cryomagnets. On the other hand, for the majority of materials used in the vacuum system of particle accelerators, electron irradiation of surfaces is known to reduce the SEY due to chemical transformations of the topmost layer [19-23]. This phenomenon is referred to as conditioning and is exploited in so-called beam-scrubbing runs of particle accelerators [9,11,24-28], where the generation of electron clouds is stimulated to modify the vacuum chamber surfaces, which in turn reduces their SEY and subsequently the electron cloud intensity.

Computer-based modeling of electron-cloud formation is a versatile and crucial tool for the design of low cloud density vacuum components and for the optimization of the operation schemes of particle accelerators [29-31]. These approaches require precise descriptions of related material properties to be used as input parameters. One of the main motivations for the present investigation is to provide such datasets. A quantity describing the effect of impinging electrons is, e.g., the SEY dependence on the primary electron kinetic energy. At present, assumptions are made for the spectral and angular distributions of the emitted electrons. However, the energy spectrum of electrons leaving the surface is composed of elastically reflected electrons (RE) and contributions from a variety of scattering and excitation processes [32-39], inelastic scattering that excites phonons, plasmons or induces interband transitions, excitation and emission of Auger electrons as well as a cascade of scattering events that lead to the emission of lowenergy true SE. Furthermore, phonon-assisted electron 
scattering leads to preferred population of states close to unoccupied band minima (critical points), where the density of states is high, or of adsorbate states. Subsequent emission from these states creates defined spectral signatures that superimpose on the featureless SE distribution [40-42]. Several empirical models were developed to describe the energy distributions of emitted SE and RE [43-47]. Moreover, Monte-Carlo simulations have proven their capability to calculate several relevant quantities, such as the inelastic mean free paths and fine structure in the energy spectra or the dependence of the SEY on the primary energy $\left(E_{\mathrm{p}}\right)$ of impinging electrons $[48,49]$. For quantitative electron-cloud simulations, especially the contribution from $\mathrm{SE}$ and RE as well as the amount of rediffused electrons with intermediate energy are of interest.

To determine these quantities experimentally, the energy dependence of SE and RE upon electron irradiation has to be characterized using energy filters. The early developments of SE emission measurements are summarized in a review article [50]. Besides the techniques that integrate the full energy spectrum of emitted electrons (SEY analysis), the energy dependence was originally analyzed by retarding-field and energy high-pass experiments [51-53]. Fully angle-integrated spectrally resolved analysis was achieved by M.P. Seah [54], while different analyzers were developed for angle-resolved measurements dedicated to electron spectroscopy. Most of these electron analyzer types exhibit a strong energy dependence of the transmission and, hence, detection efficiency. On the other hand, a four-grid retarding field analyzer (RFA) provides an almost energy-independent transmission $[55,56]$ for quantitative analyses. An RFA was previously used for the analysis of electron emission from $\mathrm{Cu}$ surfaces $[57,58]$. It enables partial angular integration that averages the contribution of the RE since the elastic reflection probability due to scattering depends on the emission angle $[59,60]$. A recent work combines such spectral analysis of SE emission from sputter-cleaned metal surfaces comparing experimental data with Monte Carlo simulations [61].

In the work presented here, special attention was paid to exposing the samples to a minimum of charge dose during the measurements that enabled the characterization of asreceived surfaces. To this end, an RFA combined with lockin amplification was used. The spectroscopic dependence of the electron emission from materials that are relevant for current and future LHC beam screens technologies was analyzed in both the unconditioned (as-received) and the fully conditioned (irradiated by an electron beam) state. The studies explored whether SE emission of low-SEY surfaces can be described by universal models or materialspecific properties play a role.

\section{EXPERIMENT}

\section{A. Apparatus}

The experiments were mainly performed at Ilmenau Technical University (ITU) in ultrahigh vacuum (UHV) $\left(10^{-9} \mathrm{~Pa}\right)$ and at room temperature. A setup originally designed for low-energy electron diffraction and Auger electron spectroscopy consisting of a four-grid RFA (SpectraLEED, Omicron) was used for acquiring energyresolved SE spectra. A resistively heated thoriated W filament yielded thermally emitted electrons that are transformed into a monochromatic electron beam with primary energies $E_{\mathrm{p}} \leq 1000 \mathrm{eV}$. The electron beam was directed perpendicular to the sample surfaces.

To achieve a minimized charge dose, which is desirable for the reduction of conditioning effects due to electron irradiation during measurements, the original RFA setup was modified. The optics of the electron source had to be operated in the mode used for low-energy electron diffraction, ensuring a sufficiently low primary electron current and a stable spot size $\left(\approx 0.7 \mathrm{~mm}^{2}\right)$ at the sample surface. At the same time, the grids of the RFA had to be operated in the Auger spectroscopy mode for energyresolved measurements with a lock-in amplifier. A further reduction of the charge dose by a defocusing of the electron beam was hampered by reaching the detection limit of the apparatus.

The modifications enabled measurements with an electron current as low as $\sim 350 \mathrm{nA}$ for $E_{\mathrm{p}}<100 \mathrm{eV}$. The current was progressively lowered with increasing primary energy and reached $100 \mathrm{nA}$ for $E_{\mathrm{p}}>500 \mathrm{eV}$, close to the lowest stable current for the present setup. For $E_{\mathrm{p}}<$ $100 \mathrm{eV}$ the conditioning effect is less effective [19]; for $E_{\mathrm{p}} \geq 100 \mathrm{eV}$ this effect increases and saturates at $E_{\mathrm{p}} \approx$ $250 \mathrm{eV}$ [19]. Table I summarizes the calculated charge doses received by the samples for the given electron currents in different $E_{\mathrm{p}}$ ranges. A total dose of $3.5 \times$ $10^{-4} \mathrm{C} / \mathrm{mm}^{2}$ was accumulated by each sample during measurements, which is nearly two orders of magnitude lower than previously reported doses [57].

Electrons scattered from the surface in a cone around the surface normal with an apex angle of $\alpha=102^{\circ}$ were collected by the RFA. Considering a $\cos \vartheta$ distribution of the emitted (secondary) electrons with $\vartheta \leq \alpha / 2$ being the angle enclosed by the emission direction and the surface normal [62], $\approx 47 \%$ of the emitted electrons are collected by the RFA. The energy distribution data, $N(E)$, were acquired with a standard lock-in amplifier method by applying a sinusoidal voltage modulation $\left(5 \mathrm{~V}_{\mathrm{pp}}, 4700 \mathrm{~Hz}\right)$ to the inner two grids of the RFA. During the measurements the

TABLE I. Charge doses accumulated by the samples during measurements for given primary energy $\left(E_{\mathrm{p}}\right)$ ranges.

\begin{tabular}{lc}
\hline \hline & Charge dose $\left(10^{-4} \mathrm{C} / \mathrm{mm}^{2}\right)$ \\
\hline$E_{\mathrm{p}}<100 \mathrm{eV}$ & 1.8 \\
$100 \mathrm{eV} \leq E_{\mathrm{p}} \leq 250 \mathrm{eV}$ & 0.7 \\
$E_{\mathrm{p}}>250 \mathrm{eV}$ & 1 \\
\hline \hline
\end{tabular}


sample was grounded. For these conditions the signal-tonoise ratio was optimized, as quantified by electron emission spectroscopy of a clean $\mathrm{Au}(111)$ surface, where the primary peak of elastically reflected electrons exhibited a full width at half maximum of $3.3 \mathrm{eV}$ at $E_{\mathrm{p}}=1008 \mathrm{eV}$ [63]. The samples exhibit a homogeneous lateral surface composition $[15,20,64]$. The onset of electron emission is defined by the work function of the sample, i.e., by the energy difference between Fermi energy $E_{\mathrm{F}}$ and vacuum level $E_{\mathrm{V}}$. For each spectrum, the linear extrapolation of the leading edge of secondary electron emission was used to determine $E_{\mathrm{V}}$ and to define $E=0 \mathrm{eV}$. This method of defining the lowest kinetic energy of electrons leaving the sample reduces the effect of different sample work functions.

Spectra were acquired with increasing $E_{\mathrm{p}}$. For each $E_{\mathrm{p}}$, the SEY was also determined with the RFA setup at ITU. To this end, the grids of the RFA were grounded. Bias voltages of $\pm 53.3 \mathrm{~V}$ were applied to the sample and the resulting currents were used to determine the SEY defined as $\left(I_{+}-I_{-}\right) / I_{+}$, where $I_{+}\left(I_{-}\right)$is the sample current to ground at positive (negative) bias voltage. The SEY was also measured with higher precision for $E_{\mathrm{p}}<1800 \mathrm{eV}$ on identically prepared twin samples in a UHV apparatus at CERN. The details of the CERN setup and the implemented experimental conditions for SEY measurements are described in detail in Ref. [20]. In the CERN setup sample bias voltages of $\pm 47.1 \mathrm{~V}$ were used. The minimum $E_{\mathrm{p}}$ at which the SEY measurements was measured at ITU was $8.6 \mathrm{eV}$, whereas at CERN the minimum $E_{\mathrm{p}}$ was $0 \mathrm{eV}$ increasing in steps of $0.1 \mathrm{eV}$. Using biased samples in principle enables SEY measurements for nearly zero kinetic energy.

The influence of magnetic stray field was minimized by carrying out the experiments in a $\mu$-metal chamber (CERN) or shielding the electron trajectories in the RFA by a $\mu$-metal cylinder (ITU). By applying the retarding bias voltage to the samples, the kinetic energy of the emitted electrons is lowered only close to the sample surface and hence the sensitivity to stray magnetic field is reduced.

\section{B. Samples}

The preparation protocols of the different samples studied here are exposed in detail in Ref. [65]. In brief, pristine polycrystalline $\mathrm{Cu}$ OFE (oxygen-free electronic grade) was used, that underwent the standard procedure for UHV cleaning at CERN, i.e., a wet-chemical detergent-based degrease procedure followed by rinsing in deionized water.

In order to generate a second type of important technical samples, these surfaces were covered with a 260 -nm-thick Ti sublayer and subsequently coated with a 100-nm-thick amorphous $\mathrm{C}$ top layer without vacuum break at CERN by magnetron sputtering from a $\mathrm{Ti}$ and a graphite target, respectively (a-C coating).

The third type of samples consists of $\mathrm{Cu}$ OFE surfaces structured by $532 \mathrm{~nm}$-picosecond laser irradiation in $\mathrm{N}_{2}$ inert atmosphere at the University of Dundee (Scotland), which resulted in a line pattern of deep grooves with a mutual separation of $\approx 24 \mu \mathrm{m}$. The details of the so-called laser-engineered surface structuring (LESS) process can be found elsewhere $[65,66]$. These samples are referred to as $\mathrm{Cu}$ LESS in the following.

After sample preparation and surface modification the samples were tightly wrapped in $\mathrm{Al}$ foil to reduce surface impurity uptake, which is of importance for the a-C coatings and the cleaned, but not passivated, $\mathrm{Cu}$ surface [15]. This aging effect is negligible for a-C coatings [15] and LESS, but could slightly affect the comparison made between $\mathrm{Cu}$ OFE samples in the two different laboratories especially for low primary energies. For this reason only the SEY of a-C-coated and LESS samples was compared. The different sample surfaces were analyzed directly after loading to the vacuum recipient (as received, ar) and after exposure to normal-incidence electron bombardment at $250 \mathrm{eV}$ with a total dose of $10^{-2} \mathrm{C} / \mathrm{mm}^{2}$ (conditioned, ce). Sample conditioning was performed by operating the electron source of the RFA (ITU) at elevated current and a flood gun (CERN) resulting in a current density of $0.41 \mu \mathrm{A} / \mathrm{mm}^{2}$ and $0.15 \mu \mathrm{A} / \mathrm{mm}^{2}$, respectively. Cu OFE samples were likewise studied after cleaning by $\mathrm{Ar}^{+}$ bombardment $(c i)$ by exposing the sample surfaces to a beam with an ion kinetic energy of $1200 \mathrm{eV}$ at a partial $\mathrm{Ar}$ pressure of $10^{-3} \mathrm{~Pa}$. The acronyms of the samples and their preparation state are listed in Table II.

TABLE II. Summary of sample acronyms.

\begin{tabular}{lcc}
\hline \hline Full Name & & Acronym \\
\hline & Samples & Cu OFE \\
Pristine polycrystalline Cu OFE & & a-C-coated \\
Amorphous C-coated $\mathrm{Cu}$ & $\mathrm{Cu}$ LESS \\
Laser-engineered surface-structured $\mathrm{Cu}$ & Preparation state & $a r$ \\
As received & & $c e$ \\
Conditioned by electron irradiation & & $c i$ \\
Cleaned by $\mathrm{Ar}^{+}$ion bombardment & & $c i$ \\
\hline \hline
\end{tabular}




\section{RESULTS AND DISCUSSION}

\section{A. Energy distribution of secondary electrons}

For each type of sample, $N(E)$ was measured at ten different values of $E_{\mathrm{p}}$. Figure 1 shows representative examples for $\mathrm{Cu}$ OFE-ci samples [63]. The data depicted in Fig. 1 are normalized to the SE maximum in order to clearly visualize the overall behavior of $N(E)$ for the wide range of primary electron energies covered and to see the relative $\mathrm{SE}$ and RE contributions to $N(E)$ at the same time. In agreement with previous findings [57] the RE contribution, which peaks at $E=E_{\mathrm{p}}$, progressively increases with a lowering of $E_{\mathrm{p}}$ and even exceeds the number of SE for $E_{\mathrm{p}} \lesssim 25 \mathrm{eV}$. This behavior was observed for all sample types investigated in this study, with the energy resolution used in this experiment. The dominance of RE at low $E_{\mathrm{p}}$ was previously reported [58] and demonstrates the high reflectivity for low-energy electrons.

The inset to Fig. 1 indicates how the fractions $\sigma$ and $\varrho$ of, respectively, $\mathrm{SE}$ and $\mathrm{RE}$ with respect to the total number of scattered electrons are extracted from $N(E)$ data. To this end, contributions of SE and RE are defined by fitting the Shirley background function [67] below the RE peak. The numerical integration of the resulting disjunct $N(E)$ datasets yields areas $A_{\mathrm{SE}}$ (gray hatched area) and $A_{\mathrm{RE}}$ (red). Fractions $\sigma$ and $\varrho$ are defined as $\sigma=A_{\mathrm{SE}} /\left(A_{\mathrm{SE}}+A_{\mathrm{RE}}\right)$ and

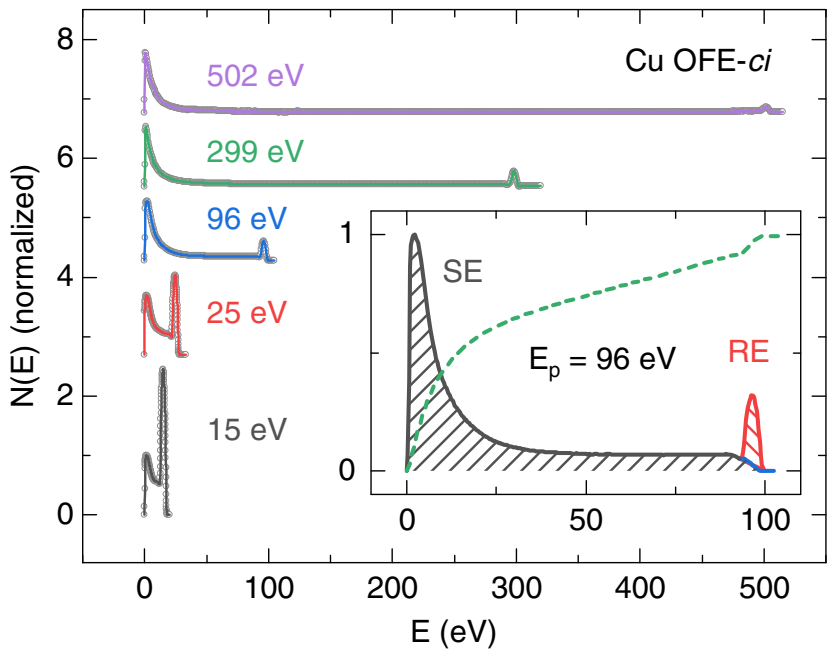

FIG. 1. Energy distribution curves, $N(E)$, of secondary and elastically reflected electrons as a function of their kinetic energy, $E$, and for the indicated primary energy, $E_{\mathrm{p}}$, of incident electrons for $\mathrm{Cu}$ OFE samples that had been cleaned by ion bombardment (ci). Raw (smoothed) data appear as dots (solid lines). The spectra are normalized by setting the SE peak height to unity. $N(E)$ data with $E_{\mathrm{p}} \geq 25 \mathrm{eV}$ are vertically offset. Inset: definition of secondary-electron (SE) and reflected-electron (RE) contributions to the total number of emitted electrons. SE and RE areas are separated by the Shirley background (blue line). Numerically integrated data, $\int N(E) \mathrm{d} E$, are normalized to unity and presented by the dashed green line.
$\varrho=A_{\mathrm{RE}} /\left(A_{\mathrm{SE}}+A_{\mathrm{RE}}\right)$. For some samples, depending on the shape of the SE peak, extraction of $\sigma$ and $Q$ was not feasible for $E_{\mathrm{p}}<10 \mathrm{eV}$, due to the overlap of the SE and RE peak. The respective data points are missing in Fig. 2 for these samples. For Cu OFE- $a r$, - ci and Cu LESS-ce, however, the SE peak was still discriminable. Spectra leading to the data of Fig. 2 can be found in the supporting information [63].

Figure 2 shows the evolution of $\sigma$ and $\varrho$ with $E_{\mathrm{p}}$ for all samples in their as-received [Fig. 2(a)] and irradiated [Fig. 2(b)] state. These datasets corroborate the aforementioned observation, i.e., the dominant contribution of RE to electron emission at low primary energies and the increasing influence of SE at higher $E_{\mathrm{p}}$. Importantly, the fraction of $\mathrm{SE}$ and RE exhibits a rather universal behavior; that is, independent of the type ( $\mathrm{Cu}$ OFE, a-C coating, $\mathrm{Cu}$ LESS) and preparation state $(a r, c e, c i)$ of the samples, $\sigma$ and $\varrho$ evolutions with $E_{\mathrm{p}}$ are approximately identical. This universal behavior contrasts the strong differences of the SEY for the samples, as discussed below. In particular, the essential independence of $\sigma$ and $\varrho$ of the specific surface texture is an important and original finding of the experiments reported here.

The Furman-Pivi model [47] represents another, more complex approach to the description of the electron emission spectra N(E). It separates the SE, RE and defines an intermediate energy range caused by backscattered

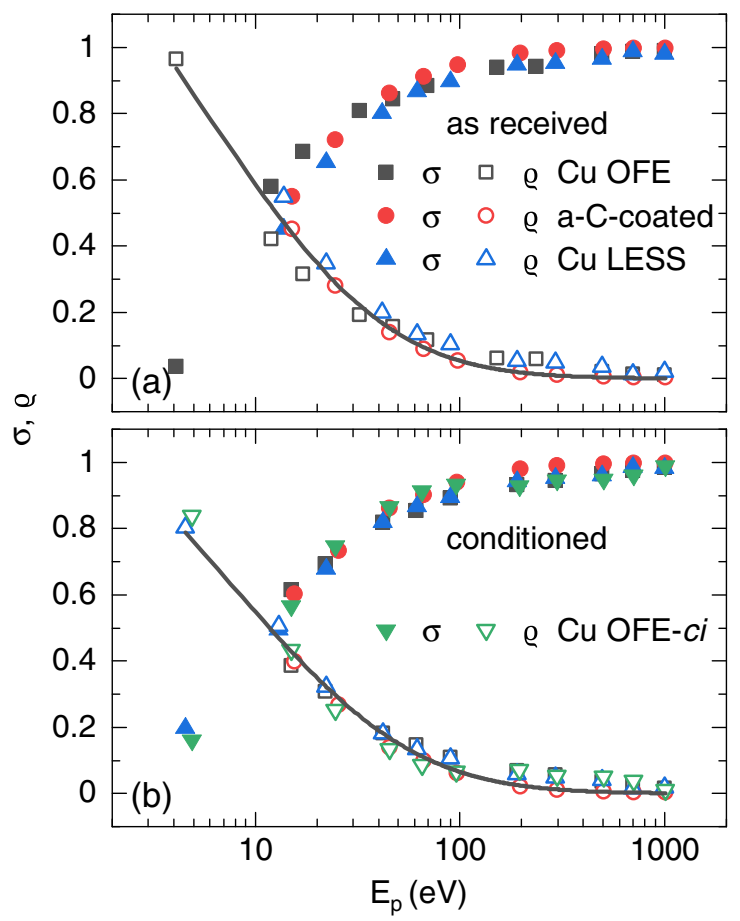

FIG. 2. Fractions $\sigma\left(E_{\mathrm{p}}\right)$ and $\varrho\left(E_{\mathrm{p}}\right)$ for (a) ar and (b) ce samples. The same symbols as in (a) are used for ce samples in (b); reversed triangles are chosen for $\mathrm{Cu}$ OFE-ci. Solid lines represent fits to the $\varrho\left(E_{\mathrm{p}}\right)$ data using Eq. (1). 
electrons. In the analysis presented here we have refrained from adding further parameters to model the obtained experimental results because a numerical representation of the experimentally observed SE and RE energy dependence can be directly implemented into electron cloud simulation codes.

Figure 2 shows that the $\varrho$ dependence on $E_{\mathrm{p}}$ may well be described by [58]

$$
\varrho\left(E_{\mathrm{p}}\right) \propto\left(\frac{\sqrt{E_{\mathrm{p}}}-\sqrt{E_{\mathrm{p}}+\varepsilon}}{\sqrt{E_{\mathrm{p}}}+\sqrt{E_{\mathrm{p}}+\varepsilon}}\right)^{2} .
$$

The physical basis of Eq. (1) is a quantum mechanical model where a plane electron wave impinges onto a negative potential step of depth $\varepsilon$. However, Eq. (1) does not fully capture the physics underlying the reflection process. Indeed, the potential depth, which is, besides a normalization factor, the only fit parameter of the model, is on the order of $100 \mathrm{eV}$ and exceeds the $\mathrm{Cu}$ work function by far [63]. An improvement of the description of electron scattering may be achieved by considering surface dipoles and image charges.

Despite the aforementioned universality, there are some differences in the $\varrho\left(E_{\mathrm{p}}\right)$ and $\sigma\left(E_{\mathrm{p}}\right)$ dependencies of the different samples. Compared to a-C-coated samples, $\mathrm{Cu}$ OFE and Cu LESS samples exhibit a slightly larger $\varrho$ for $E_{\mathrm{p}} \gtrsim 40 \mathrm{eV}$. It is likely that the a-C-coated surfaces suppress the reflection of electrons more effectively [68-70].

Simulations of electron cloud formation or heat load in accelerators rely on the empirical analytical description of experimental data. Previous calculations identified two major parameters influencing the simulated heat load, i.e., the $E_{\mathrm{p}}$ dependence of the SEY even at low $E_{\mathrm{p}}$ and the SE energy distribution [71]. Therefore, the energy distribution of secondary electrons $(E \leq 50 \mathrm{eV})$ was likewise fit using a model function [72],

$$
N(E) \propto \frac{1}{\Gamma E} \cdot \exp \left[-\frac{\ln \left(\frac{E}{\mu}\right)}{\sqrt{2} \Gamma}\right]^{2}
$$

with $\mu$ and $\Gamma$ representing parameters that influence the shape of the SE energy distribution, i.e., the energy of the emission maximum and the full width at half maximum. For $E_{\mathrm{p}}<50 \mathrm{eV}$ the spectra were cut at the onset of the RE peak to enable convergence of the fit. Figure 3(a) shows the result for Cu OFE- $c i$ samples at $E_{\mathrm{p}}=96 \mathrm{eV}$. The variation of the two fit parameters, $\mu$ and $\Gamma$, are presented in Fig. 3(b) for all probed $E_{\mathrm{p}}$. All $N(E)$ spectra may be well described by the empirical model function in Eq. (2). For decreasing $E_{\mathrm{p}} \lesssim 100 \mathrm{eV}$, both $\mu$ and $\Gamma$ increase [Fig. 3(b)]. Most likely, this increase is due to a spectroscopic fine structure of the SE peak, which leads to a broader line shape than described by Eq. (2). A fine structure was reported from SE spectroscopy of different materials attributed to
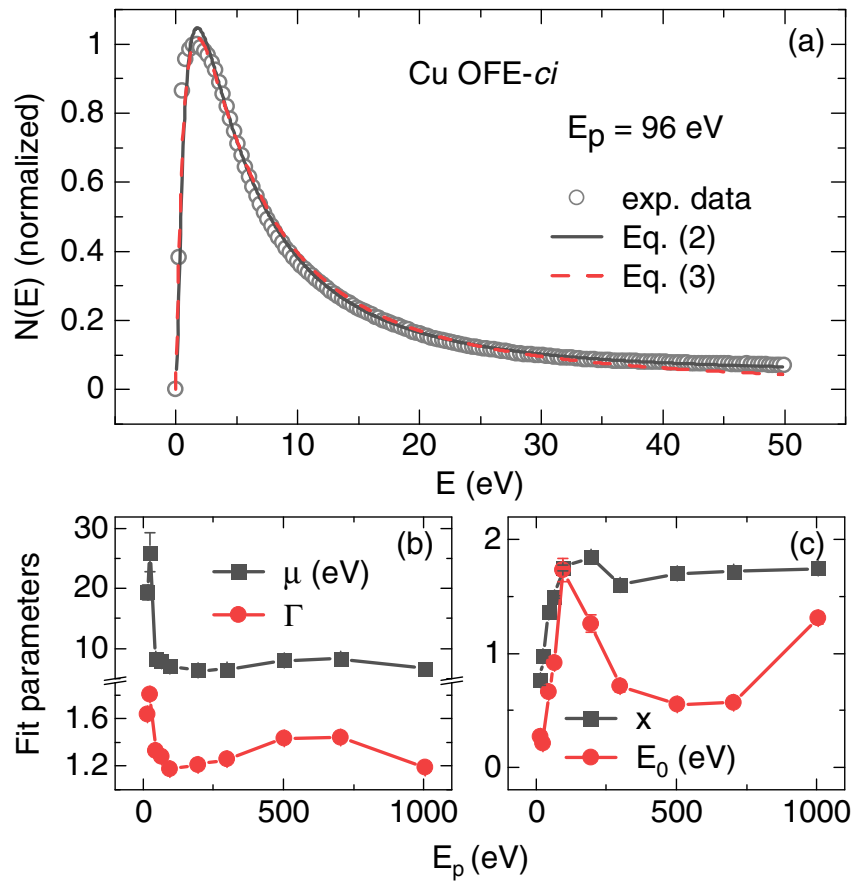

FIG. 3. Energy distribution curve, $N(E)$, of a Cu OFE sample cleaned by $\mathrm{Ar}^{+}$bombardment with focus on $\mathrm{SE}$ emission in the kinetic energy interval $0 \mathrm{eV} \leq E \leq 50 \mathrm{eV}$. (a) $N(E)$ (circles) acquired at $E_{\mathrm{p}}=96 \mathrm{eV}$. Fits to the data are depicted as solid [Eq. (2)] and dashed [Eq. (3)] lines. (b) Evolution of fit parameters $\mu, \Gamma$ [Eq. (2)] with $E_{\mathrm{p}}$. (c) Evolution of fit parameters $x$ and $E_{0}$ [Eq. (3)] with $E_{\mathrm{p}}$ for fixed $\Phi=4.5 \mathrm{eV}$.

contributions from population of critical points in the unoccupied band structure and adsorbate-induced surface resonances to the SE emission [40-42]. An increase of the energy resolution at low $E_{\mathrm{p}}$ can be excluded since the full width at half maximum of the RE peak remained essentially constant for $E_{\mathrm{p}}<300 \mathrm{eV}$.

Using a nearly-free-electron model $[43,54,73]$ the energy distribution of SE is approximated as

$$
N(E) \propto \frac{E}{(E+\Phi)^{x} \cdot\left(E+E_{0}\right)}
$$

( $\Phi$ : work function, $E_{0}$ : free-electron band edge), which holds for electron emission in any direction above the surface [54]. An exemplary fit to experimental data is presented in Fig. 3(a) as a dashed line. To fit the data, the work function $\Phi$ was set to $4.5 \mathrm{eV}$, which is a typical value for $\mathrm{Cu}$ [74]. The variation of the remaining fit parameters $x$ and $E_{0}$ with $E_{\mathrm{p}}$ is depicted in Fig. 3(c). For $E_{\mathrm{p}} \gtrsim 100 \mathrm{eV}$, $x$ levels off at $\approx 1.6$, which is in agreement with a previous report for $\mathrm{Cu}$ [54]. Fit parameter $E_{0}$, however, exhibits considerable variations with $E_{\mathrm{p}}$ and deviates from a previously reported value [54].

Due to the limitations of Eq. (3) (fixed $\Phi$, strong variation of $E_{0}$ ), Eq. (2) seems to be more suitable for the description of the data. Indeed, Eq. (2) was likewise 
used to fit the SE peak region for the other samples [63]. The extracted fit parameters exhibit an analogous behavior as observed in Fig. 3(b), i.e., they attain nearly constant values for $E_{\mathrm{p}} \gtrsim 100 \mathrm{eV}$, which are similar for all samples $-\mu$ between $\approx 5 \mathrm{eV}$ and $\approx 7 \mathrm{eV}, \Gamma$ between $\approx 0.9$ and $\approx 1.2$ - and exhibit stronger variations for $E_{\mathrm{p}} \lesssim 100 \mathrm{eV}$ [63]. Difficulties in describing $N(E)$ data at low $E_{\mathrm{p}}$ were previously reported for simulations of experimental data $[43,44,46,61,75]$. Several aspects that create a fine structure within the SE signature of the materials cannot be easily accounted for or represented in a fully quantitative manner. They can also depend on experimental conditions such as on the emission angle as well as on the crystalline properties of the samples [63]. Moreover, $N(E)$ as measured with an RFA is affected by the finite energy resolution that tends to smooth out spectroscopic fine structure. However, the resolution of a few $\mathrm{eV}$ does not modify the overall shape of $N(E)$, which can be used as input data for electron cloud simulations. The precise simulation of $N(E)$ would require the knowledge of the electronic band structure and the different faces of the polycrystalline copper surface, which is well beyond the purpose of the present work.

\section{B. Secondary-electron yield}

In order to correlate the spectral dependence of electron emission with standard energy-integrated SE measurements, which are commonly used to qualify surfaces for electron cloud mitigation capabilities, SEY $(\delta)$ measurements were performed for $E_{\mathrm{p}}<1800 \mathrm{eV}$ using identical twin samples at CERN. The data are compared to measurements performed with the RFA setup at ITU in which SEY data were measured at $E_{\mathrm{p}}$ used for spectral analyses. Figures 4(a)-4(c) show the dependence of the SEY on $E_{\mathrm{p}}$ for all samples prior to and after electron (ion) conditioning.

Owing to its lowest sensitivity to air exposure, the a-Ccoated sample can reliably be compared between the two laboratories. Therefore, Fig. 4(b) includes exemplary the comparison with the SEY measurements performed at ITU and demonstrates the overall good agreement between both experimental setups. The drop of the SEY from $\delta=1$ to $\delta \approx 0$ defines the energy at which the impinging electron can overcome the barrier of the sample vacuum level. At lower energies, the electrons are reflected from the surface which results in $\delta=1$. Above this threshold, electrons penetrate the material and with increasing $E_{\mathrm{p}}$ can excite an increasing number of SE to leave the surface. For $E_{\mathrm{p}}>\hat{E}_{\mathrm{p}}$, with $\hat{E}_{\mathrm{p}}$ the primary energy at which $\delta$ reaches its maximum, $\hat{\delta}$, the primary electrons penetrate deeper into the material and the enhancement of scattering-induced energy relaxation in the bulk leads to a lower probability of electrons to leave the surface. Qualitatively, the SEY evolves similarly for $\mathrm{Cu}$ OFE and a-C-coated samples and follows common trends of SEY curve shape [76,77],
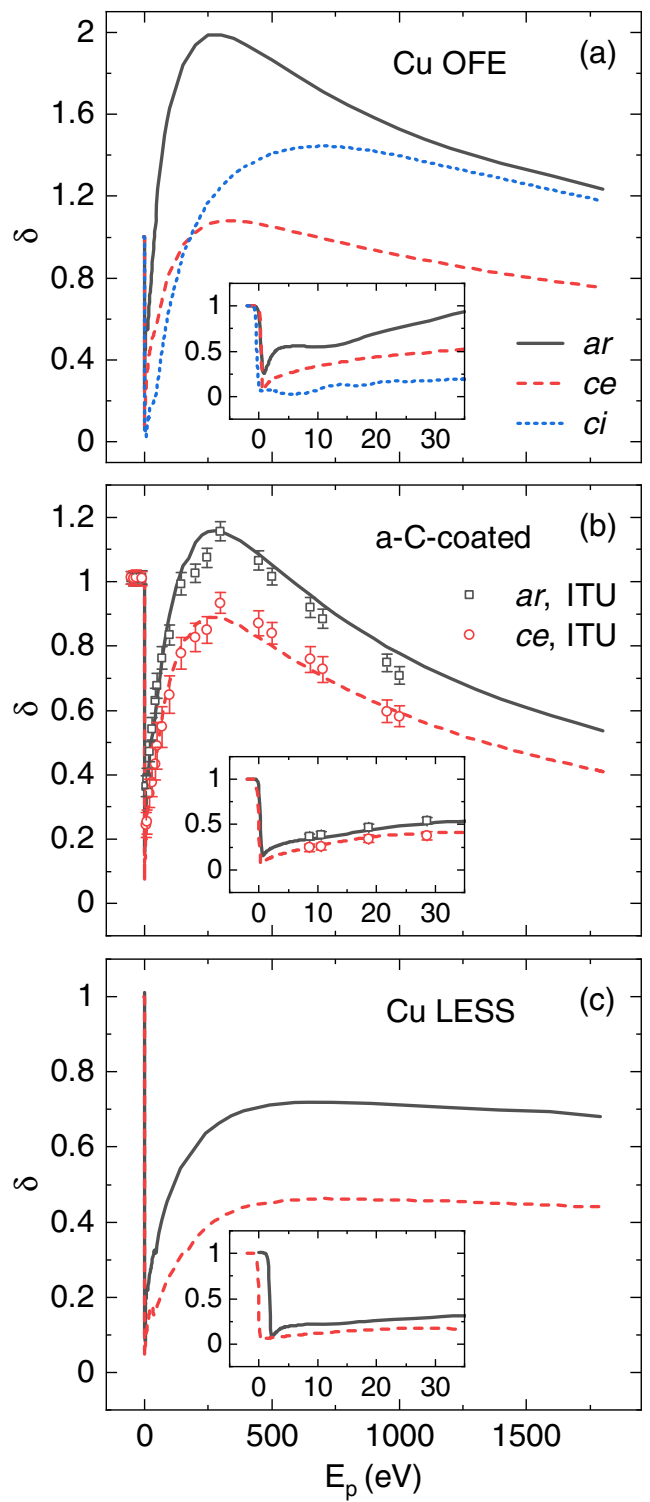

FIG. 4. Secondary-electron yield $(\delta)$ as a function of the primary energy $\left(E_{\mathrm{p}}\right)$ obtained at CERN for all types of samples studied in this work. (a) $\delta\left(E_{\mathrm{p}}\right)$ for Cu OFE surfaces. (b) Like (a), for a-C-coated samples. For comparison, ITU data are plotted as open symbols. The uncertainty margins reflect the experimental uncertainties in the measured sample current. (c) Like (a), for $\mathrm{Cu}$ LESS samples. The insets to each panel depict the low-energy $\left(0 \mathrm{eV}<E_{\mathrm{p}}<35 \mathrm{eV}\right)$ region of $\delta\left(E_{\mathrm{p}}\right)$.

with the material-dependent parameters $\hat{\delta}$ and $\hat{E}_{\mathrm{p}}$ listed in Table III. It is important to note that the properties of the characterized samples are in very good agreement with earlier SEY analyses of these different surfaces $[15,16,20,64,78]$. In particular, the SEY maximum of $\hat{\delta}=$ 1.45 for the $\mathrm{Cu}$ OFE-ci surface, Fig. 4(a), is found at $\hat{E}_{\mathrm{p}}=700 \mathrm{eV}$.

The SEY evolution of the laser-treated $\mathrm{Cu}$ surface [Fig. 4(c)], however, deviates from the $\delta\left(E_{\mathrm{p}}\right)$ behavior 
TABLE III. Summary of maximum SEY $(\hat{\delta})$ and its energy $\hat{E}_{\mathrm{p}}=E_{\mathrm{p}}(\hat{\delta})$ obtained by experiments at CERN for all types of samples in their ar and ce states.

\begin{tabular}{lccc}
\hline \hline & Cu OFE-ar;ce & a-C-coated-ar;ce & Cu LESS-ar;ce \\
\hline$\hat{\delta}$ & $1.99 ; 1.08$ & $1.16 ; 0.89$ & $0.72 ; 0.46$ \\
$\hat{E}_{\mathrm{p}}(\mathrm{eV})$ & $250 ; 350$ & $275 ; 275$ & $600 ; 700$ \\
\hline \hline
\end{tabular}

observed from the other samples. It is rather flat for $E_{\mathrm{p}} \gtrsim 300 \mathrm{eV}$, which indicates that for the nanostructured surface, different scattering mechanisms become more relevant.

Figure 4(a) shows that both electron and $\mathrm{Ar}^{+}$ion bombardment of $\mathrm{Cu}$ OFE-ar surfaces leads to a strong reduction of the SEY. The maximum SEY of the samples is reduced by $\approx 45 \%$ after electron bombardment, while $\mathrm{Ar}^{+}$ ion impact leads to a reduction of $\approx 30 \%$, in agreement with previous studies [20,78].

A strong reduction of $\hat{\delta}$ after electron irradiation is also evident for the other samples, with the SEY maximum of the a-C coating being reduced to a value slightly below unity and for the laser-treated surface even below 0.5 . This observation is in accordance with previous descriptions of electron conditioning, where beam screen material reduces its SEY due to electron cloud impact and related chemical modification of the surface $[19,22,23]$. The decrease of the SEY upon electron irradiation observed in a-C-coated samples compared to their $a r$-state shows that the latter was influenced by airborne adsorbates. Obviously, in contrast to a bare polycrystalline $\mathrm{Cu}$ surface, whose $\hat{\delta}$ is in general below the multipacting thresholds of most components of the CERN accelerator chain [30,79] only after electron conditioning, the two surface modifications-a-C coating and surface laser treatment- do not require conditioning to efficiently suppress the electron cloud effect. The prevention of any avalanche effect of electron emission allows in theory an efficient electron cloud mitigation, which is especially required for the high-luminosity operation of the LHC starting in the upcoming years [80] and for the recently approved electron-ion collider (EIC) $[81,82]$.

The low-energy SEY [insets to Fig. 4(a)-(c)] exhibits distinct features that result from the sample electronic structure. The surface composition of the individual materials as well as possible surface adsorbates due to air exposure contribute to these features. Because of a low electron inelastic mean free path $(<1 \mathrm{~nm})$ in this energy range, low-energy SEY is particularly sensitive to the outermost composition of the surface. For $\mathrm{Cu}$ and other metal surfaces $[20,83,84]$ as well as for carbon-based materials $[85,86]$ these aspects were previously discussed. Further detailed studies focusing on the description of these features are the topic of forthcoming research.

\section{CONCLUSION}

Using a four-grid RFA combined with lock-in detection enables the energy-resolved acquisition of SE emission spectra with low charge dose of the sample. A universal behavior of SE ratios with the primary energy of impinging electrons is observed for pristine, a-C-coated and lasertreated $\mathrm{Cu}$ samples, which is nearly identical for asreceived and conditioned surfaces. The SEY is lowest for the laser-structured surfaces for all primary energies.

\section{ACKNOWLEDGMENTS}

The authors thank A. R. Granadeiro Costa and P. Costa Pinto, Technology Department (TE-VSC-SCC), CERN for the preparation of the amorphous carbon coated samples. The laser treated samples were kindly provided by D. Bajek, S. Wackerow and A. Abdolvand, School of Science \& Engineering, University of Dundee, Dundee, Scotland, UK.

[1] A. A. Manalio, K. Burin, and G. M. Rothberg, More efficient channel electron multiplier by coating of the cone with a high secondary electron yield material, Rev. Sci. Instrum. 52, 1490 (1981).

[2] S. X. Tao, H. W. Chan, and H. Van der Graaf, Secondary electron emission materials for transmission dynodes in novel photomultipliers: A review, Materials 9, 1017 (2016).

[3] H. Seiler, Secondary electron emission in the scanning electron microscope, J. Appl. Phys. 54, R1 (1983).

[4] T. Suganuma, Measurement of surface topography using SEM with two secondary electron detectors, J. Electron Microsc. 34, 328 (1985).

[5] B. L. Thiel and M. Toth, Secondary electron contrast in low-vacuum/environmental scanning electron microscopy of dielectrics, J. Appl. Phys. 97, 051101 (2005).

[6] N. Balcon, D. Payan, M. Belhaj, T. Tondu, and V. Inguimbert, Secondary electron emission on space materials: Evaluation of the total secondary electron yield from surface potential measurements, IEEE Trans. Plasma Sci. 40, 282 (2012).

[7] S. T. Lai, The role of surface condition in the yields of secondary electrons, backscattered electrons, and photoelectrons from spacecraft, IEEE Trans. Plasma Sci. 41, 3492 (2013).

[8] M. Izawa, Y. Sato, and T. Toyomasu, The Vertical Instability in a Positron Bunched Beam, Phys. Rev. Lett. 74, 5044 (1995).

[9] O. Domínguez, K. Li, G. Arduini, E. Métral, G. Rumolo, F. Zimmermann, and H. M. Cuna, First electron-cloud studies at the Large Hadron Collider, Phys. Rev. Accel. Beams 16, 011003 (2013).

[10] R. Cimino and T. Demma, Electron cloud in accelerators, Int. J. Mod. Phys. A 29, 1430023 (2014).

[11] Y. Suetsugu, K. Shibata, T. Ishibashi, K. Kanazawa, M. Shirai, S. Terui, and H. Hisamatsu, First commissioning of the SuperKEKB vacuum system, Phys. Rev. Accel. Beams 19, 121001 (2016). 
[12] W. Fischer, M. Blaskiewicz, J. M. Brennan, H. Huang, H.-C. Hseuh, V. Ptitsyn, T. Roser, P. Thieberger, D. Trbojevic, J. Wei, S. Y. Zhang, and U. Iriso, Electron cloud observations and cures in the Relativistic Heavy Ion Collider, Phys. Rev. Accel. Beams 11, 041002 (2008).

[13] G. Arduini et al., High Luminosity LHC: Challenges and plans, J. Instrum. 11, C12081 (2016).

[14] C. Y. Vallgren, G. Arduini, J. Bauche, S. Calatroni, P. Chiggiato, K. Cornelis, P. C. Pinto, B. Henrist, E. Métral, H. Neupert, G. Rumolo, E. Shaposhnikova, and M. Taborelli, Amorphous carbon coatings for the mitigation of electron cloud in the CERN Super Proton Synchrotron, Phys. Rev. Accel. Beams 14, 071001 (2011).

[15] P. C. Pinto, S. Calatroni, H. Neupert, D. Letant-Delrieux, P. Edwards, P. Chiggiato, M. Taborelli, W. Vollenberg, C. Yin-Vallgren, J. Colaux, and S. Lucas, Carbon coatings with low secondary electron yield, Vacuum 98, 29 (2013).

[16] R. Valizadeh, O. B. Malyshev, S. Wang, S. A. Zolotovskaya, W. A. Gillespie, and A. Abdolvand, Low secondary electron yield engineered surface for electron cloud mitigation, Appl. Phys. Lett. 105, 231605 (2014).

[17] R. Valizadeh, O. Malyshev, S. Wang, T. Sian, M. Cropper, and N. Sykes, Reduction of secondary electron yield for Ecloud mitigation by laser ablation surface engineering, Appl. Surf. Sci. 404, 370 (2017).

[18] D. Bajek, S. Wackerow, D. A. Zanin, L. Baudin, K. Bogdanowicz, E. G.-T. Valdivieso, S. Calatroni, B. D. Girolamo, M. Sitko, M. Himmerlich, M. Taborelli, P. Chiggiato, and A. Abdolvand, Role of surface microgeometries on electron escape probability and secondary electron yield of metal surfaces, Sci. Rep. 10, 250 (2020).

[19] R. Cimino, M. Commisso, D. R. Grosso, T. Demma, V. Baglin, R. Flammini, and R. Larciprete, Nature of the Decrease of the Secondary-Electron Yield by Electron Bombardment and its Energy Dependence, Phys. Rev. Lett. 109, 064801 (2012).

[20] V. Petit, M. Taborelli, H. Neupert, P. Chiggiato, and M. Belhaj, Role of the different chemical components in the conditioning process of air exposed copper surfaces, Phys. Rev. Accel. Beams 22, 083101 (2019).

[21] C. Scheuerlein, M. Taborelli, N. Hilleret, A. Brown, and M. A. Baker, An AES study of the room temperature conditioning of technological metal surfaces by electron irradiation, Appl. Surf. Sci. 202, 57 (2002).

[22] B. Henrist, N. Hilleret, C. Scheuerlein, M. Taborelli, and G. Vorlaufer, The variation of the secondary electron yield and of the desorption yield of copper under electron bombardment: Origin and impact on the conditioning of the LHC, in Proceedings of the 8th European Particle Accelerator Conference, Paris, 2002 (EPS-IGA and CERN, Geneva, 2002), pp. 2553-2555, http://cds.cern.ch/record/566863.

[23] M. Nishiwaki and S. Kato, Electron stimulated gas desorption from copper material and its surface analysis, Appl. Surf. Sci. 169-170, 700 (2001).

[24] Y. Suetsugu, K. Shibata, T. Ishibashi, H. Fukuma, M. Tobiyama, J. Flanagan, E. Mulyani, M. Shirai, S. Terui, K.-i. Kanazawa, and H. Hisamatsu, Achievements and problems in the first commissioning of superKEKB vacuum system, J. Vac. Sci. Technol. A 35, 03 E103 (2017).
[25] G. Iadarola, H. Bartosik, T. Bohl, B. Goddard, G. Kotzian, K. Li, L. Mether, G. Rumolo, M. Schenk, E. Shaposhnikova, and M. Taborelli, Detailed studies of beam induced scrubbing in the CERN-SPS, in Proc. 6th International Particle Accelerator Conference (IPAC'15), International Particle Accelerator Conference No. 6 (JACoW, Geneva, Switzerland, 2015), pp. 3908-3910, https://doi.org/10.18429/JACoW-IPAC2015-THPF091.

[26] O. S. Brüning, J. Poole, P. Collier, P. Lebrun, R. Ostojic, S. Myers, and P. Proudlock, LHC Design Report, CERN Yellow Reports: Monographs (CERN, Geneva, 2004), https://doi.org/10.5170/CERN-2004-003-V-1.

[27] S. Y. Zhang, W. Fischer, H. Huang, and T. Roser, Beam Scrubbing for RHIC Polarized Proton Operation, BNL Report (Brookhaven National Laboratory, Upton, NY, 2003), https://doi.org/10.2172/1061716.

[28] J. Jimenez, Mini-Workshop on SPS Scrubbing run (CERN, Geneva, 2002).

[29] T. Demma, S. Petracca, F. Ruggiero, G. Rumolo, and F. Zimmermann, Maps for electron cloud density in Large Hadron Collider dipoles, Phys. Rev. Accel. Beams 10, 114401 (2007).

[30] G. Iadarola, Electron cloud studies for CERN particle accelerators and simulation code development, Ph.D. thesis, University of Naples, Italy, 2014, https://cds.cern .ch/record/1705520.

[31] A. Romano, O. Boine-Frankenheim, X. Buffat, G. Iadarola, and G. Rumolo, Electron cloud buildup driving spontaneous vertical instabilities of stored beams in the Large Hadron Collider, Phys. Rev. Accel. Beams 21, 061002 (2018).

[32] J. J. Lander, Auger peaks in the energy spectra of secondary electrons from various materials, Phys. Rev. 91, 1382 (1953).

[33] L. A. Harris, Analysis of materials by electron-excited Auger electrons, J. Appl. Phys. 39, 1419 (1968).

[34] S. Tougaard and P. Sigmund, Influence of elastic and inelastic scattering on energy spectra of electrons emitted from solids, Phys. Rev. B 25, 4452 (1982).

[35] S. Tougaard and I. Chorkendorff, Differential inelastic electron scattering cross sections from experimental reflection electron-energy-loss spectra: Application to background removal in electron spectroscopy, Phys. Rev. B 35, 6570 (1987).

[36] A. Jablonski, Elastic scattering and quantification in AES and XPS, Surf. Interface Anal. 14, 659 (1989).

[37] R. Shimizu and D. Ze-Jun, Monte Carlo modelling of electron-solid interactions, Rep. Prog. Phys. 55, 487 (1992).

[38] W. S. M. Werner, Electron transport in solids for quantitative surface analysis, Surf. Interface Anal. 31, 141 (2001).

[39] G. Gergely, Elastic backscattering of electrons: Determination of physical parameters of electron transport processes by elastic peak electron spectroscopy, Prog. Surf. Sci. 71, 31 (2002).

[40] R. Kollath, Zur Energieverteilung der Sekundärelektronen II. Meßergebnisse und Diskussion, Ann. Phys. (Leipzig) 436, 357 (1947). 
[41] J. Schäfer, R. Schoppe, J. Hölzl, and R. Feder, Experimental and theoretical study of the angular resolved secondary electron spectroscopy (ARSES) for W(100) in the energy range $0 \leq E \leq 20 \mathrm{eV}$, Surf. Sci. 107, 290 (1981).

[42] R. Feder, B. Awe, and E. Tamura, Theory of the fine structure in secondary electron emission from $\mathrm{Cu}(001)$ c $(2 \times 2)$-Cl, Surf. Sci. 157, 183 (1985).

[43] P. A. Wolff, Theory of secondary electron cascade in metals, Phys. Rev. 95, 56 (1954).

[44] G. F. Amelio, Theory for the energy distribution of secondary electrons, J. Vac. Sci. Technol. 7, 593 (1970).

[45] J. Schou, Transport theory for kinetic emission of secondary electrons from solids by electron and ion bombardment, Nucl. Instrum. Methods 170, 317 (1980).

[46] T. Kaneko, Energy distribution of secondary electrons emitted from solid surfaces under electron bombardment, Surf. Sci. 237, 327 (1990).

[47] M. A. Furman and M. T. F. Pivi, Probabilistic model for the simulation of secondary electron emission, Phys. Rev. Accel. Beams 5, 124404 (2002).

[48] Z.-J. Ding and R. Shimizu, Monte Carlo study of backscattering and secondary electron generation, Surf. Sci. 197, 539 (1988).

[49] K. Ohya, A. Harada, J. Kawata, and K. Nishimura, Monte Carlo simulation of yield and energy distribution of secondary electrons emitted from metal surfaces, Jpn. J. Appl. Phys. 35, 6226 (1996).

[50] R. Kollath, Sekundärelektronen-Emission fester Körper bei Bestrahlung mit Elektronen, in Electron-Emission Gas Discharges I / Elektronen-Emission Gasentladungen I, Encyclopedia of Physics / Handbuch Der Physik, edited by W. B. Nottingham, R. H. Good, E. W. Müller, R. Kollath, G. L. Weissler, W. P. Allis, L. B. Loeb, A. von Engel, and P. F. Little (Springer, Berlin, Heidelberg, 1956), pp. 232-303, https://doi.org/10.1007/978-3-642-458446_3.

[51] A. Becker, Über die Rückdiffusion, Reflexion und Sekundärstrahlerregung langsamer Kathodenstrahlen, Ann. Phys. (Leipzig) 383, 253 (1925).

[52] K. Sixtus, Untersuchungen über Sekundäremission, Ann. Phys. (Leipzig) 395, 1017 (1929).

[53] H. W. Langenwalter, Über Rückdiffusion und Sekundärstrahlerregung langsamer Kathodenstrahlen an dünnen Metallschichten, Ann. Phys. (Leipzig) 416, 273 (1935).

[54] M. P. Seah, Slow electron scattering from metals: I. The emission of true secondary electrons, Surf. Sci. 17, 132 (1969).

[55] B. Gruzza, P. Bondot, A. Porte, C. Jardin, and G. Gergely, The transmission of the electron analyzers determined by elastic peak electron spectroscopy, Acta Phys. Pol. A 81, 159 (1992).

[56] A. Alkafri, Y. Ichikawa, R. Shimizu, and K. Goto, Transmission measurement of the absolute CMA; simulation and experiments, J. Surf. Anal. 14, 2 (2007).

[57] R. Cimino and I. R. Collins, Vacuum chamber surface electronic properties influencing electron cloud phenomena, Appl. Surf. Sci. 235, 231 (2004).

[58] R. Cimino, I. R. Collins, M. A. Furman, M. Pivi, F. Ruggiero, G. Rumolo, and F. Zimmermann, Can
Low-Energy Electrons Affect High-Energy Physics Accelerators?, Phys. Rev. Lett. 93, 014801 (2004).

[59] T. Oguri, H. Ishioka, H. Fukuda, and M. Irako, Angular distribution of scattered electron and medium energy electron spectroscopy for metals, J. Phys. Soc. Jpn. 55, 414 (1986).

[60] W. S. M. Werner, I. S. Tilinin, and M. Hayek, Angular distribution of electrons reflected elastically from noncrystalline solid surfaces, Phys. Rev. B 50, 4819 (1994).

[61] M. Azzolini, M. Angelucci, R. Cimino, R. Larciprete, N. M. Pugno, S. Taioli, and M. Dapor, Secondary electron emission and yield spectra of metals from Monte Carlo simulations and experiments, J. Phys. Condens. Matter 31, 055901 (2019).

[62] H. Bruining, Physics and Applications of Secondary Electron Emission (Pergamon Science, London, 1962), https://doi.org/10.1016/C2013-0-01633-7.

[63] See Supplemental Material at http://link.aps.org/ supplemental/10.1103/PhysRevAccelBeams.23.103101 for full $N(E)$ data acquired from a $\mathrm{Au}(111)$ surface, that was used for calibration purposes; for $N(E)$ data obtained for Cu OFE-ar (Fig. S1), Cu OFE-ce (Fig. S2), Cu OFE-ci (Fig. S3), a-C-coated-ar (Fig. S4) and a-C-coated-ce (Fig. S5) samples, Cu LESS-ar (Fig. S6), Cu LESS-ce (Fig. S7) with a variety of primary electron energies; for individual fits of Eq. (1) to $\varrho$ obtained from $\mathrm{Cu}$ OFE, a-C-coated surfaces and Cu LESS samples in their ar and ci state (Fig. S8); for the variation of the fit parameters $\mu$ and $\Gamma$ for $\mathrm{Cu}$ OFE-ce, a-C-coated-ce and $\mathrm{Cu}$ LESS-ce samples (Fig. S9).

[64] S. Calatroni, E. G.-T. Valdivieso, A. T. P. Fontenla, M. Taborelli, H. Neupert, M. Himmerlich, P. Chiggiato, D. Bajek, S. Wackerow, and A. Abdolvand, Optimization of the secondary electron yield of laser-structured copper surfaces at room and cryogenic temperature, Phys. Rev. Accel. Beams 23, 033101 (2020).

[65] S. Calatroni, M. Arzeo, S. Aull, M. Himmerlich, P. C. Pinto, W. Vollenberg, B. Di Girolamo, P. Cruikshank, P. Chiggiato, D. Bajek, S. Wackerow, and A. Abdolvand, Cryogenic surface resistance of copper: Investigation of the impact of surface treatments for secondary electron yield reduction, Phys. Rev. Accel. Beams 22, 063101 (2019).

[66] R. Salemme, V. Baglin, S. Calatroni, P. Chiggiato, B. Di Girolamo, E. G.-T. Valdivieso, B. Jenninger, L. PreverLoiri, M. Sitko, S. Wackerow, and A. Abdolvand, First beam test of Laser Engineered Surface Structures (LESS) at cryogenic temperature in CERN SPS accelerator, J. Phys. Conf. Ser. 1067, 082017 (2018).

[67] D. A. Shirley, High-resolution X-ray photoemission spectrum of the valence bands of gold, Phys. Rev. B 5, 4709 (1972).

[68] S. Tanuma, C. J. Powell, and D. R. Penn, Calculations of electron inelastic mean free paths. IX. Data for 41 elemental solids over the $50 \mathrm{eV}$ to $30 \mathrm{keV}$ range, Surf. Interface Anal. 43, 689 (2011).

[69] H.-J. Fitting, E. Schreiber, J.-C. Kuhr, and A. von Czarnowski, Attenuation and escape depths of low-energy electron emission, J. Electron Spectrosc. Relat. Phenom. 119, 35 (2001). 
[70] C. Bouchard and J. Carette, The surface potential barrier in secondary emission from semiconductors, Surf. Sci. 100, 251 (1980).

[71] L. Bitsikokos, G. Iadarola, L. Sabato, and P. Dijkstal, Secondary Emission Models in E-Cloud Buildup Simulations: From the Lab to the Code, 6th Workshop on Electron-Cloud Effects (ECLOUD'18), La Biodola Bay, Isola d'Elba, Italy, 2018, https://agenda.infn.it/event/ 13351/contributions/18937/.

[72] J. J. Scholtz, D. Dijkkamp, and R. W. A. Schmitz, Secondary electron emission properties, Philips J. Res. 50, 375 (1996).

[73] H. W. Streitwolf, Zur Theorie der Sekundärelektronenemission von Metallen Der Anregungsprozeß, Ann. Phys. (Leipzig) 458, 183 (1959).

[74] J. Hölzl and F. K. Schulte, Work function of metals, in Solid Surface Physics, Vol. 85, edited by J. Hölzl, F. K. Schulte, and H. Wagner (Springer, Berlin, Heidelberg, 1979), pp. 1-150, https://doi.org/10.1007/BFb0048919.

[75] M. Dapor, A Monte Carlo investigation of secondary electron emission from solid targets: Spherical symmetry versus momentum conservation within the classical binary collision model, Nucl. Instrum. Methods Phys. Res., Sect. B 267, 3055 (2009).

[76] E. M. Baroody, A theory of secondary electron emission from metals, Phys. Rev. 78, 780 (1950).

[77] R. G. Lye and A. J. Dekker, Theory of secondary emission, Phys. Rev. 107, 977 (1957).

[78] R. Larciprete, D. R. Grosso, M. Commisso, R. Flammini, and R. Cimino, Secondary electron yield of $\mathrm{Cu}$ technical surfaces: Dependence on electron irradiation, Phys. Rev. Accel. Beams 16, 011002 (2013).
[79] G. Iadarola and G. Rumolo, Electron cloud in the CERN accelerators (PS, SPS, LHC), AIP Conf. Proc. C 1206051, 19 (2013).

[80] G. Apollinari, I. B. Alonso, O. Brüning, P. Fessia, M. Lamon, L. Rossi, and L. Tavian, High-Luminosity Large Hadron Collider (HL-LHC) Technical Design Report V. 0.1, CERN Yellow Reports: Monographs, Vol. 4/ 2017, Report No. CERN-2017-007-M (CERN, 2017), https://doi.org/10.23731/CYRM-2017-004.

[81] V. Ptitsyn et al., eRHIC, a future electron-ion collider at BNL, in Proceedings of the 9th European Particle Accelerator Conference, Lucerne, 2004 (EPS-AG, Lucerne, 2004), pp. 923-925 [http://accelconf.web.cern.ch/ AccelConf/e04/].

[82] An Electron-Ion Collider Study, edited by J. Beebe-Wang, 2019 (unpublished), https://wiki.bnl.gov/eic/upload/EIC .Design.Study.pdf.

[83] L. A. Gonzalez, M. Angelucci, R. Larciprete, and R. Cimino, The secondary electron yield of noble metal surfaces, AIP Adv. 7, 115203 (2017).

[84] R. Cimino, L. A. Gonzalez, R. Larciprete, A. Di Gaspare, G. Iadarola, and G. Rumolo, Detailed investigation of the low energy secondary electron yield of technical $\mathrm{Cu}$ and its relevance for the LHC, Phys. Rev. Accel. Beams 18, 051002 (2015).

[85] A. Bellissimo, G. M. Pierantozzi, A. Ruocco, G. Stefani, O. Y. Ridzel, V. Astašauskas, W. S. Werner, and M. Taborelli, Secondary electron generation mechanisms in carbon allotropes at low impact electron energies, J. Electron Spectrosc. Relat. Phenom. 241, 146883 (2020).

[86] L. A. Gonzalez, R. Larciprete, and R. Cimino, The effect of structural disorder on the secondary electron emission of graphite, AIP Adv. 6, 095117 (2016). 